\title{
Increased BDNF levels and NTRK2 gene association suggest a disruption of BDNF/TrkB signaling in autism
}

C. T. Correia ${ }^{\dagger, t, \S}$ A. M. Coutinho ${ }^{\dagger}$, A. F. Sequeira ${ }^{\dagger, t^{\prime}, \S}$, I. G. Sousa ${ }^{\dagger}$, L. Lourenço Venda $^{\dagger}$, J. P. Almeida", R. L. AbreuT, C. Lobo", T. S. Miguel**, J. Conroy ${ }^{\dagger+}$, L. Cochrane ${ }^{\dagger \dagger}$, L. Gallagher ${ }^{\dagger t}$, M. Gill ${ }^{\dagger+}$, S. Ennis ${ }^{\ddagger \ddagger}$ G. G. Oliveira ${ }^{\prime}$ and A. M. Vicente ${ }^{*, t, \neq, \S}$

${ }^{\dagger}$ Instituto Gulbenkian de Ciência, Oeiras, ${ }^{\ddagger}$ Departamento de Promoção da Saúde e Doenças Crónicas, Instituto Nacional de Saúde Dr. Ricardo Jorge, Lisboa, § Center for Biodiversity, Functional \& Integrative Genomics, Lisboa, "Centro de Desenvolvimento da Criança, Hospital Pediátrico de Coimbra, Coimbra, "** Direcção Regional de Educação da Região Centro, Coimbra, Portugal, ${ }^{+\dagger}$ Neuropsychiatric Genetics Group, IMM, Trinity Centre for Health Sciences, St. James Hospital, Dublin,

${ }^{\ddagger}$ School of Medicine and Medical Science, University College, Dublin, Ireland

${ }^{*}$ Corresponding authors: A. M. Vicente, Instituto Nacional de Saúde Dr. Ricardo Jorge, Av. Padre Cruz, 1649-016 Lisboa, Portugal. E-mail: astrid.vicente@insa.min-saude.pt

The brain-derived neurotrophic factor (BDNF), a neurotrophin fundamental for brain development and function, has previously been implicated in autism. In this study, the levels of BDNF in platelet-rich plasma were compared between autistic and control children, and the role of two genetic factors that might regulate this neurotrophin and contribute to autism etiology, $B D N F$ and NTRK2, was examined. We found that BDNF levels in autistic children $(n=146)$ were significantly higher $(t=6.82 ; P<0.0001)$ than in control children $(n=50)$ and were positively correlated with platelet serotonin distribution $(r=0.22 ; P=0.004)$. Heritability of BDNF was estimated at $30 \%$ and therefore candidate genes BDNF and NTRK2 were tested for association with BDNF level distribution in this sample, and with autism in 469 trio families. Genetic association analysis provided no evidence for BDNF or NTRK2 as major determinants of the abnormally increased BDNF levels in autistic children. A significant association with autism was uncovered for six single nucleotide polymorphisms (SNPs) $\left[0.004\left(Z_{(1 \mathrm{df})}=2.85\right)<P<0.039\left(Z_{(1 \mathrm{df})}=2.06\right)\right]$ and multiple haplotypes $\left[5 \times 10^{-4}\left(\chi_{(3 \mathrm{df})}=17.77\right)<P<\right.$ $\left.0.042\left(\chi_{(9 \mathrm{df})}=17.450\right)\right]$ in the NTRK2 gene. These results do not withstand correction for multiple comparisons, however, reflect a trend toward association that supports a role of $N T R K 2$ as a susceptibility factor for the disorder. Genetic variation in the BDNF gene had no impact on autism risk. By substantiating the previously observed increase in BDNF levels in autistic children in a larger patient set, and suggesting a genetic association between NTRK2 and autism, this study integrates evidence from multiple levels supporting the hypothesis that alterations in BDNF/tyrosine kinase B (TrkB) signaling contribute to an increased vulnerability to autism.

Keywords: Autism, BDNF, genetic association, heritability, NTRK2

Received 30 March 2010, revised 28 June 2010 and 7 July 2010, accepted for publication 7 July 2010

Autism is a neurodevelopmental syndrome characterized by deficits in social interaction, impaired communication and restricted and stereotyped behaviors (Folstein \& RosenSheidley 2001). Although the etiology of autism is unclear, it may result from an abnormal development of the central nervous system (CNS) with alterations of neuronal organization, cortical connectivity and neurotransmitter pathways, and with a significant genetic influence (Pardo \& Eberhart 2007).

Multiple lines of evidence directly or indirectly suggest an involvement in autism of the brain-derived neurotrophic factor (BDNF), a member of the neurotrophin family, which plays a pivotal role in the development and plasticity of the brain. Brain-derived neurotrophic factor has a trophic effect for specific neuronal populations during development and in the mature brain, influencing neuronal survival, morphology, differentiation and synaptic strength (Lang et al. 2004). Like other neurotrophins, BDNF acts through transmembrane receptors that modulate diverse biological actions, namely the specific high-affinity tyrosine kinase B (TrkB) receptor, encoded by the NTRK2 gene, and the non-specific low-affinity receptor p75. Direct evidence supporting an involvement of the BDNF/TrkB signaling pathway in autism comes from several studies in small population samples showing that BDNF levels in the blood, serum and brain are increased in autistic children compared with normal controls (Connolly et al. 2006; Miyazaki et al. 2004; Nelson et al. 2001; Perry et al. 2001). Genetic studies provide additional support for this hypothesis: in a family-based association study, specific BDNF single nucleotide polymorphism (SNP) haplotypes were associated with autism (Nishimura et al. 2007), and a whole-genome scan with dense SNP coverage, performed by the Autism Genome Project (AGP) consortium in a large set of autism families, identified a linkage peak with genome-wide significance on chromosome 11p12-13, where the BDNF gene maps (AGP et al. 2007). Variants of the BDNF and NTRK2 genes have been associated with several neuropsychiatric disorders, including bipolar disorder, schizophrenia, depression, attention deficit and hyperactivity 
disorder (ADHD) and obsessive compulsive disorder (OCD) (Alonso et al. 2008; Fanous et al. 2004; Neves-Pereira et al. 2002; Ribases et al. 2008). Other observations provide an indirect evidence for a role of BDNF in autism. Psychostimulants and some antidepressant drugs commonly used for autism treatment, including tricyclic antidepressants and selective serotonin reuptake inhibitors (SSRIs), modulate the expression of BDNF and its specific receptor TrkB (Duman 1998). Conditional Ntrk2 knockout mice (TrkB-CRE), showing reduced synaptic plasticity, display impaired behavioral flexibility, a trait that is frequently disrupted in individuals with autism (Vyssotski et al. 2002). Other mouse models in which the Ntrk2 gene has been disrupted show hyperlocomotion, stereotyped behaviors and cognitive impairments, all behavioral problems typically associated with autism (Zorner et al. 2003).

These previous observations led us to hypothesize that BDNF/TrkB signaling alterations in the brain, with consequences to CNS structure and function, may contribute to an increased vulnerability to autism. To test this hypothesis, we first investigated whether BDNF levels in platelet-rich plasma (PRP), the main source of circulating BDNF, are altered in autistic patients relative to healthy control subjects in the same age range, and are correlated with sex, age or medication. We then estimated the heritability of this trait and tested the BDNF and NTRK2 genes for association with BDNF PRP levels distribution and with autism.

\section{Methods}

\section{Population sample and clinical assessments}

The population sample in this study included 301 autistic patients lage range from 2 to 18 years, mean age of 6.3 years, male:female ratio 5.27:1) and their parents, originating from Portugal and recruited at Hospital Pediátrico de Coimbra. In this population, or subsets, we measured BDNF and serotonin levels, estimated heritability and tested the association of specific genetic variants with BDNF levels. The analysis of genetic association with autism was performed in a total of 469 parent-child trios, including the 301 Portuguese trios and a second set of 168 families recruited through schools, advocacy groups and healthcare professionals in Ireland. Patients were evaluated using standard research diagnostic procedures for autism, and met the Autism Diagnostic Interview-Revised (ADI-R) (Lord et al. 1994) criteria for autism and the Autism Diagnostic Observation Schedule-Generic (ADOS-G) (Lord et al. 2000) criteria for autism or autism spectrum disorder (ASD). Developmental or intellectual quotients were determined using the Ruth Griffiths Mental Scale II or the Wechsler Intelligence Scale for Children (WISC), and all patients had a developmental level or intellectual quotient above 25 and mental age above 18 months. Participants were mainly of Caucasian origin. Individuals with known medical causes of autism were excluded, e.g. tuberous sclerosis, fragile $X$ syndrome, chromosomal anomalies, metabolic disorders, extreme prematurity, congenital rubella. Brain-derived neurotrophic factor and serotonin levels were measured in a subset of the Portuguese patients. Thirty of these subjects were medicated with at least one of the following classes of drugs: antiepileptics, neuroleptics, SSRI, anxiolytics, psychostimulants. For BDNF and serotonin quantification, 54 healthy control children (age range from 4 months to 18 years, mean age of 7.5 years, male:female ratio $1.75: 1)$ were recruited from orthopedic wards at the Hospital Pediátrico de Coimbra, where they were drawing blood for the purpose of small surgical procedures, and were otherwise healthy and free of the above drug classes (Coutinho et al. 2004). All participants or their legal representatives signed an informed written consent and ethical approval was obtained from the Ethics Committee at Hospital
Pediátrico de Coimbra and Eastern Regional Health Authority, Child and Adolescent Psychiatry Ethics Committee, for the Portuguese and Irish populations, respectively.

\section{BDNF quantification}

Brain-derived neurotrophic factor levels were measured in PRP isolated from blood as previously described (Coutinho et al. 2004) and stored frozen. Platelets are the major storage location for circulating BDNF (Fujimura et al. 2002), which is released into plasma by freezing and thawing cycles of the plasma sample. Quantification of BDNF was carried out by enzyme-linked immunosorbent assay (ELISA) using the BDNF Emax Immunoassay System kit (Promega, Madison, WI, USA), in 146 patients (age range from 2 to 15 years, mean age of 7.1 years), 88 parents and 50 healthy control children (age range from 4 months to 18 years, mean age of 7.5 years) from the Portuguese sample. Platelet-rich plasma dilution of $1 / 200$ with sample buffer supplied by the kit was required to obtain adequate concentrations for quantification. The absorbance was recorded at $450 \mathrm{~nm}$ using a SpectraMax 340PC microplate reader (Molecular Devices, Sunnyvale, CA, USA). All BDNF measurements were performed in duplicate, and duplicate determinations of absorbance with a coefficient of variance $>10 \%$ were excluded. To control for the interassay variance, patient and control samples were always assayed in the same plate, and PRP samples from a group of four subjects, with BDNF concentrations spread across the standard curve, were included in every plate as internal controls. The coefficient of variation for this group in all plates was $<15 \%$. In 99 subjects with BDNF measurements, platelet 5-hydroxytrypiamine 5-HT had been previously quantified by highperformance liquid chromatography (HPLC) (Coutinho et al. 2004).

\section{Candidate gene genotyping}

To ensure full genetic coverage, we used information on the CEPH panel from the public release 22 of the HapMap project dataset and HAPLOVIEW to select BDNF and NTRK2 tagSNPs according to the default algorithm $\left[r^{2}>0.8\right.$; minor allele frequency (MAF) $\left.>5 \%\right]$. In the BDNF region, 15 SNPs, including the 11 tagSNPs, were genotyped, and in the NTRK2 region, genotyping was carried out for 71 tagSNPs and 7 additional SNPs.

Genotyping was carried out using the Sequenom ${ }^{\circledR}$ MassARRAY technology (Sequenom ${ }^{\circledR}$, San Diego, CA, USA). Polymerase chain reaction (PCR) and MassExtend primers were designed using ASSAY DESIGN Version 3.1 software. The PCR primers were used to amplify $7.5 \mathrm{ng}$ of genomic DNA using standard conditions for MassARRAY genotyping. The chip was scanned using a mass spectrometry workstation (Sequenom MassARRAY System) and the resulting spectra were analyzed using the Sequenom SPECTROTYPER-RT Version 3.4 software. HapMap samples were included in the genotyping assays. All markers with a call rate below $80 \%$ ( 1 in the BDNF gene and 3 in the NTRK2 gene) and not in Hardy-Weinberg equilibrium at the $5 \%$ significance level (2 in the BDNF gene and 10 in the NTRK2 gene) were excluded from further analysis. After the quality control, 12 markers in BDNF and 65 in NTRK2 were tested for association with autism and BDNF levels.

\section{Statistical analysis}

The correlation between BDNF levels and other parameters that could influence BDNF levels was ascertained using Spearman's correlation coefficient or non-parametric tests. To evaluate the differences in BDNF levels between autistic and control children, a linear regression analysis was carried out, including the variables, as potential confounding factors, observed to be correlated with BDNF levels at a significance level of 0.1 (age and medication status). The SPSS software was used for the calculations. Inconsistent Mendelian inheritance, non-paternity or other typing errors in the trio datasets were identified using PEDCHECK (O'Connell \& Weeks 1998). Heritability of BDNF levels was estimated using the variance-components (VC) analysis procedure implemented in Quantitative Transmission Disequilibrium Test (OTDT) software Version 2.5.1. With OTDT, estimates of the heritability of the trait are obtained by comparing 
a model including an environmental variance with a model including polygenic and environmental variances (Abecasis et al. 2000).

To test the association of marker alleles with BDNF levels, we performed a quantitative transmission disequilibrium test implemented in the OTDT software, following a square-root transformation of the data to obtain a normal distribution, which is required by the software. An orthogonal model of association was considered, including VC (individual and nuclear family environment and a polygenic component).

Family-based tests of association with autism were performed using the FBAT program for single markers (Horvath et al. 2001). Pairwise linkage disequilibrium (LD) between the various markers, based on $D^{\prime}$ values, and haplotype block structures were evaluated by HAPLOVIEW software version 3.2 (Barrett et al. 2005). Sliding window two- to four-marker haplotypes were tested for association using the TRANSMIT program (Clayton \& Jones 1999). Uncorrected $P<0.05$ was regarded as significant. Multiple testing correction was carried out using the Bonferroni method or permutation analysis. To calculate the power of our transmission disequilibrium test (TDT), the PBAT program was used (Lange \& Laird 2002). We assumed an additive model, a disease prevalence of 0.001 with a targeted significance level of 0.05 and marker and disease allele frequencies of 0.2 .

\section{Results}

\section{PRP BDNF levels in autistic vs. control children}

The distribution of BDNF PRP levels in 146 autistic and 50 control Portuguese children is shown in Fig. 1. Mean BDNF levels in autistic patients and in control children were $40.44 \pm 13.87 \mathrm{ng} / \mathrm{ml}$ and $23.26 \pm 12.34 \mathrm{ng} / \mathrm{ml}$, respectively. We found that $25 \%$ of the autistic children have increased BDNF levels, defined as values above the control mean +2 SD $(47.95 \mathrm{ng} / \mathrm{ml})$. We found no correlation of BDNF levels with several clinical, demographic and experimental variables that could influence BDNF levels, namely gen$\operatorname{der}(Z=-0.419 ; P=0.675)$, age (Spearman $r=0.139 ; P=$ $0.094)$, medication status $\left(\chi^{2}(1)=3.614 ; P=0.057\right)$, developmental level (assessed using the Ruth Griffiths Mental Developmental Scale II) (Spearman $r=-0.072 ; P=0.418$ ), autism severity (assessed using the Childhood Autism Rating

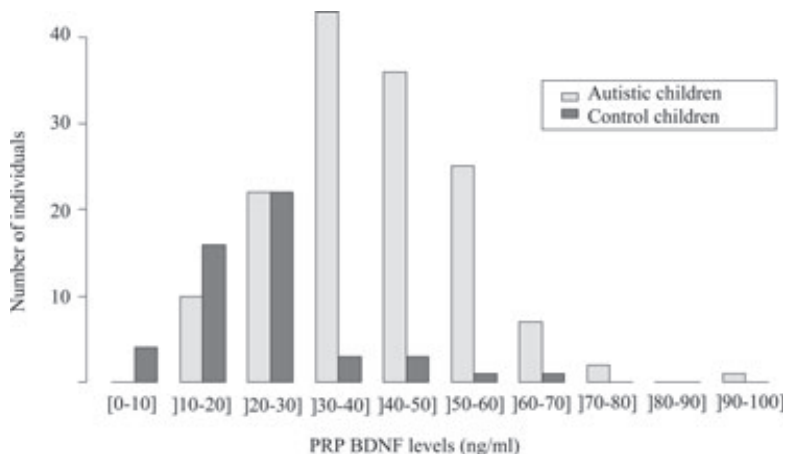

Figure 1: Distribution of BDNF levels in the platelet-rich plasma of autistic and control children. The number of individuals is represented on the Y-axis, plotted in a decimal metric scale, and on the $\mathrm{X}$-axis is shown each PRP-PDNF levels interval (measured in $\mathrm{ng} / \mathrm{ml}$ ). The bars in dark grey represent the control sample, whereas the bars in light grey represent the individuals with autism.
Scale CARS) (Spearman $r=0.016 ; P=0.851$ ) and date of sample collection $\left(\chi^{2}(3)=1.169 ; P=0.761\right)$. Linear regression analysis showed significant effects of medication and age $(t=2.84 ; P=0.005$ for age; $t=2.58 ; P=0.011$ for medication) and, after adjusting for these confounding factors, we found a highly significant difference in BDNF levels between autistic and control children $(t=6.82 ; P<0.0001)$.

Because platelet hyperserotonemia has consistently been associated with autism and several findings indicate that there is a positive, reciprocal interaction between 5-HT and BDNF (Duman 1998; Mattson et al. 2004), with 5-HT stimulating the expression of BDNF and BDNF enhancing the growth and survival of 5-HT neurons (Zetterstrom et al. 1999), we analyzed the correlation between PRP BDNF and platelet serotonin levels in the affected children. The distribution of PRP BDNF in this population sample showed a significant positive correlation with platelet serotonin levels (Spearman $r=0.222 ; P=0.004$ ).

\section{Heritability of BDNF levels and investigation of candidate genes}

The polygenic heritability of PRP BDNF was estimated at $29.7 \%$, indicating that almost one third of the total variance in BDNF levels can be explained by a genetic component. This observation prompted us to investigate the role of two candidate genes involved in the regulation of BDNF, BDNF and NTRK2, which might contribute to its increase in about $25 \%$ of the autistic children in this study population.

Using a family-based association test for quantitative traits, the OTDT, we could not find any evidence for an association of the BDNF or NTRK2 genes with the distribution of PRP BDNF.

We further conducted an association analysis with autism in a larger trio set. The tested population sample consisted of 469 trios with $87 \%$ powered to detect association at a significance level of $5 \%$. Results of FBAT showed a significant transmission disequilibrium for several SNPS in the NTRK2 gene: rs1187321, located 5' of the gene, rs1211166, rs893584, rs1187352 located in intron 5-6 and rs4144550 in intron 15-16 and a nominal association with the rs4578034 marker in intron 22-23 $(0.004<P<0.039$, uncorrected

Table 1: Transmission disequilibrium test results of significantly associated NTRK2 markers with autism in the 469 trio dataset

\begin{tabular}{|c|c|c|c|c|c|c|}
\hline Marker & $\begin{array}{c}\text { Over } \\
\text { transmitted } \\
\text { allele }\end{array}$ & Afreq & Fam\# & $S-E(S)$ & $Z$ & $P$-value \\
\hline Rs1187321 & $A$ & 0.79 & 211 & 23.0 & 2.85 & 0.004 \\
\hline Rs1211166 & A & 0.79 & 208 & 21.5 & 2.70 & 0.007 \\
\hline Rs893584 & $\mathrm{G}$ & 0.58 & 271 & 23.5 & 2.43 & 0.015 \\
\hline Rs1187352 & C & 0.67 & 268 & 26.0 & 2.74 & 0.006 \\
\hline Rs4144550 & G & 0.87 & 156 & 16.0 & 2.41 & 0.016 \\
\hline Rs4578034 & C & 0.43 & 279 & 20.0 & 2.06 & 0.039 \\
\hline
\end{tabular}

afreq, allele frequency; $E(S)$, expected value under the null hypothesis; fam\#, the number of informative families; $S$, test statistic; $Z, S$ normalized.

The $P$-values shown are not corrected for multiple testing. 


\section{Correia et al.}

Table 2: Significant NTRK2 haplotype association results for the 469 trio dataset

\begin{tabular}{|c|c|c|c|c|c|c|c|c|c|}
\hline Haplotype & Location & $\chi^{2}(\mathrm{df})$ & Global $P$ & $\begin{array}{c}\text { Over } \\
\text { transmitted }\end{array}$ & $\chi^{2}(1)$ & $P$-value & $\begin{array}{c}\text { Under } \\
\text { transmitted }\end{array}$ & $\chi^{2}(1)$ & $P$-value \\
\hline 1_3 & 5'Region & $11.218(3)$ & 0.0106 & - & - & - & $\mathrm{CT} / \mathrm{TT}$ & $4.453 / 6.604$ & $0.0350 / 0.010$ \\
\hline 3_4 & 5' Region/intron 5-6 & $10.220(3)$ & 0.0168 & - & - & - & $\mathrm{TC} / \mathrm{TT}$ & $4.140 / 6.211$ & $0.0420 / 0.0130$ \\
\hline 4_5 & Intron 5-6 & $13.088(3)$ & 0.0045 & - & - & - & $\mathrm{CG} / \mathrm{TG}$ & $4.427 / 9.087$ & $0.0350 / 0.0026$ \\
\hline 8_9 & Intron 5-6 & $10.058(3)$ & 0.0181 & GC & 5.837 & 0.0157 & AT & 7.910 & 0.0049 \\
\hline 9_10 & Intron 5-6/intron 7-8 & $17.765(3)$ & 0.0005 & CA & 7.076 & 0.0078 & TA/TG & $6.296 / 8.445$ & $0.0121 / 0.0037$ \\
\hline 43_44 & Intron 19-20 & $11.640(3)$ & 0.0087 & - & - & - & $A C$ & 6.397 & 0.0114 \\
\hline 61_62 & Intron 22-23 & $8.379(3)$ & 0.0388 & - & - & - & TG & 4.510 & 0.0337 \\
\hline 63_64 & Intron 22-23 & $13.355(3)$ & 0.0039 & GC & 5.668 & 0.0173 & AT & 9.090 & 0.0026 \\
\hline 64_65 & Intron 22-23 & $8.761(3)$ & 0.0326 & - & - & - & TT/TC & $4.280 / 4.106$ & $0.0386 / 0.0430$ \\
\hline $1 \_3 \_4$ & 5' Region/intron 5-6 & $16.170(6)$ & 0.0129 & - & - & - & СТT/TTC & $4.815 / 6.695$ & $0.0280 / 0.010$ \\
\hline 3_4_5 & 5' Region/intron 5-6 & $14.925(6)$ & 0.0208 & - & - & - & TCG/TTG & $4.395 / 8.917$ & $0.0360 / 0.003$ \\
\hline 4_5_6 & Intron 5-6 & $16.387(6)$ & 0.0118 & - & - & - & TGT & 8.863 & 0.0029 \\
\hline 1_3_4_5 & 5' Region/intron 5-6 & $13.361(6)$ & 0.0376 & - & - & - & CTTG/TTTG & $7.534 / 20.230$ & $0.006 / 6.9 \times 10^{-06}$ \\
\hline 3_4_5_6 & 5' Region/intron 5-6 & $17.450(9)$ & 0.0421 & - & - & - & TTGT & 8.814 & 0.0030 \\
\hline 1_3_4_5_6 & 5' Region/intron 5-6 & $13.881(5)$ & 0.0164 & - & - & - & CTTGT/TTTGT & $7.410 / 8.695$ & $0.0065 / 0.0032$ \\
\hline 8_9_10 & Intron 5-6/intron 7-8 & $22.287(7)$ & 0.0023 & GCA & 5.268 & 0.0217 & ATA/ATG & $5.545 / 9.387$ & $0.0185 / 0.0022$ \\
\hline 61_62_63 & Intron 22-23 & $17.955(7)$ & 0.0122 & - & - & - & TAA & 9.850 & 0.0017 \\
\hline 62_63_64 & Intron 22-23 & $19.027(7)$ & 0.0081 & - & - & - & AAT/GGT & $9.083 / 4.381$ & $0.0026 / 0.0360$ \\
\hline 63_64_65 & Intron 22-23 & $17.412(5)$ & 0.0038 & GCC & 5.003 & 0.0253 & ATC/GTT & $9.063 / 4.228$ & $0.0026 / 0.040$ \\
\hline
\end{tabular}

The SNP numbers used here are as shown in Fig. 2. The $P$-values shown are not corrected for multiple testing.

$P$-values; Table 1). These results did not remain significant after correction for multiple comparisons.

Evidence of association with autism was also found for two-, three-, four- and five-marker haplotypes containing the associated SNPs in the 5' region and intron 5-6 (Table 2), with the strongest association observed for haplotype rs1189752-rs1659409 $\left[\chi^{2}(3)=17.77 ; P=0.0005\right.$, uncorrected $P$-value]. Significant two- and three-marker haplotypes including SNPs not individually associated were also identified in intron 22-23, with haplotype rs4271046-rs4578034rs12001219 being the strongest finding $\left[\chi^{2}(5)=17.41\right.$; $P=0.0038$, uncorrected $P$-value]. No haplotype association remained significant after correction for multiple comparisons. Pair-wise $D^{\prime}$ values across the association regions were determined (Fig. 2) and the LD pattern shows that markers in the 5'-untranslated region (5'-UTR) and intron 5-6 are likely signaling the same risk allele in this region. Markers and haplotypes associated in intron 22-23 region, located in an LD block that spans $17 \mathrm{~kb}$, are possibly signaling a second autism risk allele. The results therefore define two possible main regions of susceptibility to autism, one from $5^{\prime}$ to intron 7-8 and other in intron 22-23.

The tested trio set consists of two independently recruited populations, of Irish $(n=168)$ and Portuguese $(n=301)$ origins. Because genetic ancestry of the two samples is considerably distinct, we more closely inspected the patterns of SNP association in each population. When we analyzed the two populations separately, we found that in the Portuguese dataset, the association was already significant for five of the six SNPs associated in the combined trio sample. The Irish trio population, although underpowered to detect association independently, generally showed the same pattern of allelic transmissions. The magnitude of association of all the significant SNPs was increased or maintained in the combined trio set (data not shown). These observations therefore indicate that the association results are driven by both populations.

There was no evidence for association of BDNF markers with autism in the population tested.

\section{Discussion}

In the present study, we examined the involvement of the BDNF signaling pathway in autism using various approaches. First, PRP BDNF levels were compared in autistic and control children, in the largest population sample thus far reported. The observed BDNF increase in $25 \%$ of our autistic patients extended previous evidence for quantitative BDNF anomalies in autism, obtained in smaller cohorts and in diverse tissues (Connolly et al. 2006; Hashimoto et al. 2006; Katoh-Semba et al. 2007; Miyazaki et al. 2004; Nelson et al. 2001; Perry et al. 2001). A significant correlation with age was detected, corroborating previous studies showing that serum or plasma BDNF levels in rats (Karege et al. 2002) and healthy human subjects (Katoh-Semba et al. 2007; Lommatzsch et al. 2005; Nelson et al. 2006) are markedly altered by age. A significant correlation of BDNF levels with medication was also observed, in agreement with studies that show that several drugs commonly used for pharmacotherapy in autism, such as antipsychotics, antidepressants or antiepileptics, affect the expression of BDNF in several brain regions both in rats and humans (Biagini et al. 2001; Duman 1998; Fumagalli et al. 2003). The low number of medicated patients precluded the analysis of the effect of different drug groups separately. No significant difference in BDNF levels between males and females was observed, in agreement with previous reports (Katoh-Semba et al. 2007; Lommatzsch et al. 2005; Nelson et al. 2006). 

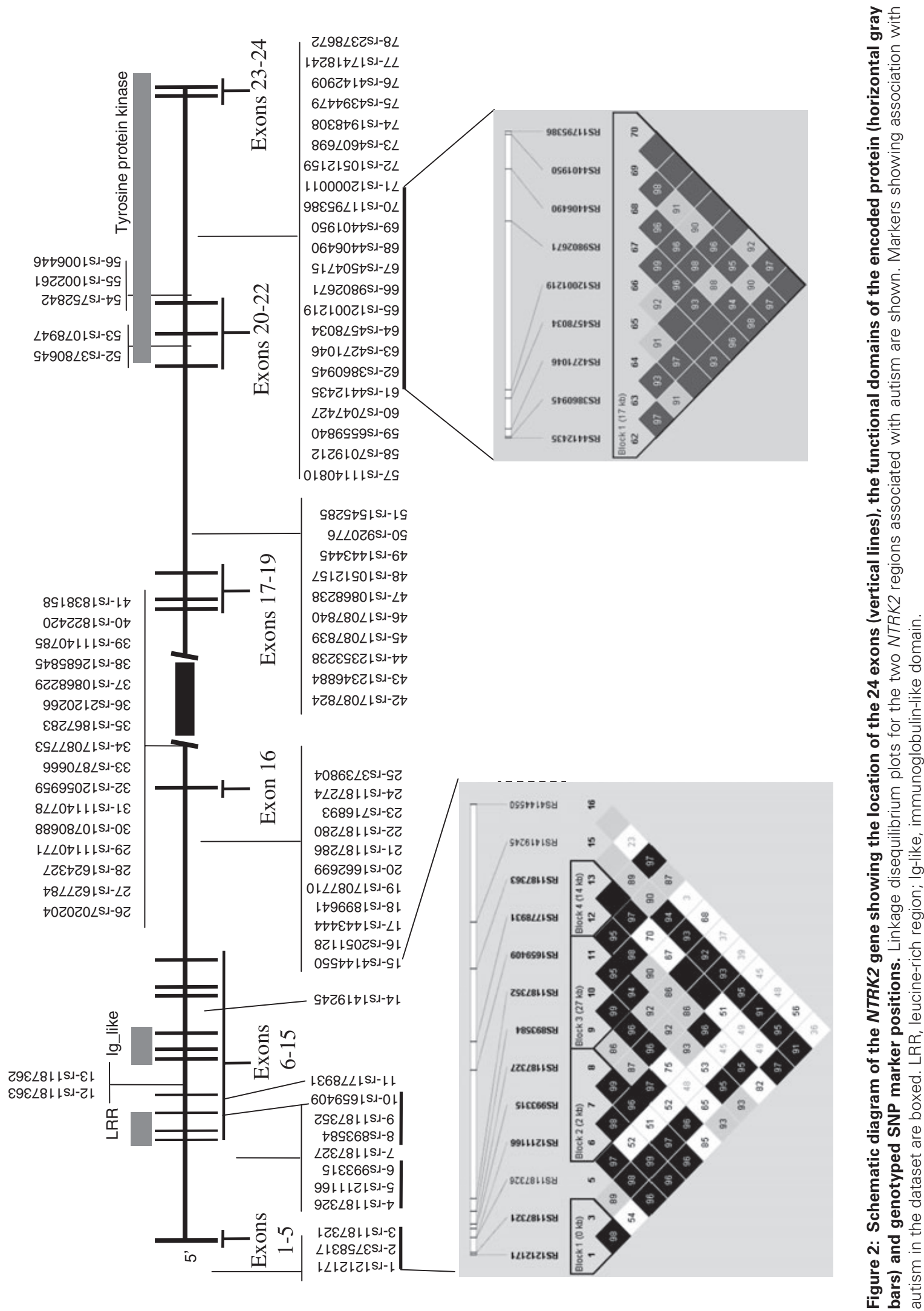


\section{Correia et al.}

The strong positive correlation found between BDNF PRP levels and platelet serotonin levels is consistent with the described crossregulation between BDNF and serotonin (5-HT) (Mattson et al. 2004) and with the platelet hyperserotonemia found in subgroups of autistic individuals (Coutinho et al. 2004).

The origin of increased BDNF in PRP, and its relation to brain BDNF levels, is not clear. Although BDNF is highly concentrated in the nervous system, it is also found in other tissues including blood, where it is essentially stored in platelets and thought to be involved in platelet activation and associated events (Fujimura et al. 2002). This is not unexpected, as platelets and neurons develop from a common embryonic precursor in the neural crest (Pearse 1980). Karege et al. (2002) have shown that the variation profile of BDNF protein in serum and brain is similar during postnatal development in rats, with a positive correlation between serum and cortical BDNF levels (Karege et al. 2002). In humans, the developmental curve of serum BDNF has been shown to be similar to that of the neurotrophin in the rat neocortex (Katoh-Semba et al. 2007; Nelson et al. 2006). These observations suggest that BDNF levels in the blood may reflect BDNF levels in the brain. This parallelism between brain and blood variation is similar to what has been postulated for the serotonergic system. A fully functional serotonin pathway is required for platelet aggregation, and many of the molecules involved in this system are present both in brain and platelets. Platelet hyperserotonemia has been a hallmark of autism, with variation in serotonin-related genes associated with this trait thought to functionally disturb the serotonin pathway both in the CNS and in platelets. Although the origin and function of BDNF in blood are less clear, our finding of a strong correlation between platelet serotonin and BDNF levels suggests a parallel variation between serotonin and BDNF, both in brain and in the periphery. On the other hand, the estimation of BDNF heritability close to $30 \%$ supported the hypothesis that the observed BDNF increase in autistic individuals results, at least in part, from a genetic primary defect that may have an effect on BDNF signaling pathways in every tissue where it is present, namely blood and brain.

None of the tested markers in the NTRK2 and BDNF genes, fully covering genetic variation in these regions, were associated with BDNF levels, including the described BDNF functional polymorphism Val66Met (rs6265), associated with abnormal intracellular trafficking and regulated secretion of BDNF (Egan et al. 2003). The lack of association of the Val66Met polymorphism with BDNF levels was consistent with previous reports in other neuropsychiatric conditions or healthy subjects (Duncan et al. 2008; Mercader et al. 2007; Trajkovska et al. 2007; Yu et al. 2008), while previous studies examining the association of BDNF with autism report discrepant results (Nishimura et al. 2007). We suggest that the increase in levels of BDNF is not due to variants of the BDNF gene or its receptor, which means that other genes that affect BDNF transcription and secretion are likely responsible for this change. However, we cannot rule out the hypothesis that association with BDNF levels could not be detected because of the low power of the tested sample.

The current study provided for the first time evidence for an association between the NTRK2 gene and autism.
Two main regions of susceptibility in the NTRK2 gene were identified (from the $5^{\prime}$ end to intron 7-8 and in intron 2223), and should have high priority in searching for functional variants. Exons 6, 7 and 8 together encode a leucine-rich domain that has a crucial role in ligand binding, while exons neighboring intron 22-23 encode a tyrosine kinase domain (Stoilov et al. 2002). A bioinformatics investigation of the putative functional effects of the associated SNP in the $5^{\prime}$ region, using GENOMATIX, showed that this SNP lies in the core of a sequence with high similarity to the matrix of the homeodomain transcription factors (HOMF) family and of the factors with moderate activity to homeodomain consensus sequence HOXF family, and that allele T in this SNP disrupts the binding of these factors. Many of these homeobox genes, such as PITX1, HOXA1 and the DLX family, have been associated with autism (Ingram et al. 2000; Philippi et al. 2007) and, remarkably, a recent functional study found that NTRK2 is a transcriptional target of DLX2 during mouse retinal development (de Melo et al. 2007).

The genetic association results were not significant after correction for multiple comparisons. Working with a relatively small population sample, this result is not unexpected, as the increase in risk conveyed by common genetic factors will likely be subtle for autism, as is the case for most complex disorders. As there is no consensus regarding the most appropriate method for multiple testing correction, it is generally accepted that the replication of a genetic association in independent populations is considered the best evidence of a true association. We observe in this study that the two datasets included, with distinct genetic ancestries, show the same pattern of allelic transmissions for the significantly associated SNPs and haplotypes, indicating that the association in the combined sample was driven by both populations. Further genetic studies in larger datasets are required to fully validate this hypothesis.

In the absence of definitive statistical evidence or a method for appropriately correcting for multiple testing, approaches that integrate evidence from multiple levels, including biological observations, are also valuable tools for assessing the validity of results. In this study, we provide two lines of evidence which, taken together, suggest that an alteration in the BDNF/TrkB signaling is involved in autism etiology, because of the presence of risk genetic variants of the receptor, or to a genetically determined increased level of the ligand. Because we found no evidence for a role of NTRK2 in determining the abnormally increased BDNF, we suggest that these two processes are separate. Other genetic factors would be expected to contribute to the increase in BDNF, while NTRK2 risk variants operate downstream in this pathway. Interestingly, there is increasing evidence that several syndromic disorders co-morbid with autism, rare mutations and common variants associated with autism converge on dysfunctional signaling of the Phosphatidylinositol-3kinase (PI3K)-Akt pathway, one of the three signaling pathways activated by BDNF (Levitt \& Campbell 2009). A genetically determined disruption of BDNF/TrkB signaling in autism may therefore occur at different levels: in the availability of the ligand, in the catalytic or binding affinity of the receptor or in alterations of the signaling cascades. Abnormalities in various neurotransmitter systems have been implicated in autism, 
including the glutamatergic, GABAergic and serotonergic systems (Lam et al. 2006), all of which are regulated by the BDNF/TrkB signaling system. It is therefore a plausible hypothesis that altered BDNF/TrkB signaling may be an upstream event contributing to abnormalities in these neurotransmitter systems, frequently reported in autism.

In conclusion, our study provides evidence for a dysregulation of the BDNF/TrkB signaling system in autism. We report an increase in BDNF PRP levels in 25\% of the autistic children in this population sample, the largest tested thus far. We further provide evidence that the NTRK2 gene is a susceptibility factor for autism, although we did not find any evidence for a role of the NTRK2 or BDNF genes in mediating the BDNF increase in autistic children. We therefore propose that a disruption of the BDNF/TrkB signaling pathway is associated with autism and, consistent with the etiological heterogeneity expected for autism, can occur at different levels, including the availability of the ligand, the catalytic or binding affinity of the receptor and the subsequent signaling cascades.

\section{References}

Abecasis, G.R., Cardon, L.R. \& Cookson, W.O. (2000) A general test of association for quantitative traits in nuclear families. Am J Hum Genet 66, 279-292.

AGP, Szatmari, P., Paterson, A.D. et al. (2007) Mapping autism risk loci using genetic linkage and chromosomal rearrangements. Nat Genet 39, 319-328

Alonso, P., Gratacos, M., Menchon, J.M., Saiz-Ruiz, J., Segalas, C., Baca-Garcia, E., Labad, J., Fernandez-Piqueras, J., Real, E., Vaquero, C., Perez, M., Dolengevich, H., Gonzalez, J.R., Bayes, M., de Cid, R., Vallejo, J. \& Estivill, X. (2008) Extensive genotyping of the BDNF and NTRK2 genes define protective haplotypes against obsessive-compulsive disorder. Biol Psychiatry 63, 619-628.

Barrett, J.C., Fry, B., Maller, J. \& Daly, M.J. (2005) Haploview: analysis and visualization of LD and haplotype maps. Bioinformatics 21, 263-265.

Biagini, G., Avoli, M., Marcinkiewicz, J. \& Marcinkiewicz, M. (2001) Brain-derived neurotrophic factor superinduction parallels antiepileptic-neuroprotective treatment in the pilocarpine epilepsy model. J Neurochem 76, 1814-1822.

Clayton, D. \& Jones, H. (1999) Transmission/disequilibrium tests for extended marker haplotypes. Am J Hum Genet 65, 1161-1169.

Connolly, A.M., Chez, M., Streif, E.M., Keeling, R.M., Golumbek, P.T., Kwon, J.M., Riviello, J.J., Robinson, R.G., Neuman, R.J. \& Deuel, R.M. (2006) Brain-derived neurotrophic factor and autoantibodies to neural antigens in sera of children with autistic spectrum disorders, Landau-Kleffner syndrome, and epilepsy. Biol Psychiatry 59, 354-363.

Coutinho, A.M., Oliveira, G., Morgadinho, T., Fesel, C., Macedo, T.R., Bento, C., Marques, C., Ataide, A., Miguel, T., Borges, L. \& Vicente, A.M. (2004) Variants of the serotonin transporter gene (SLC6A4) significantly contribute to hyperserotonemia in autism. Mol Psychiatry 9, 264-271.

de Melo, J., Zhou, Q.-P., Zhang, Q., Zhang, S., Fonseca, M., Wigle, J. \& Eisenstat, D. (2007) Dlx2 homeobox gene transcriptional regulation of Trkb neurotrophin receptor expression during mouse retinal development. Nucleic Acids Res 36, 872-884.

Duman, R.S. (1998) Novel therapeutic approaches beyond the serotonin receptor. Biol Psychiatry 44, 324-335.

Duncan, L.E., Hutchison, K.E., Carey, G. \& Craighead, W.E. (2008) Variation in brain-derived neurotrophic factor (BDNF) gene is associated with symptoms of depression. J Affect Disord 115, $215-219$.
Egan, M.F., Kojima, M., Callicott, J.H., Goldberg, T.E., Kolachana, B.S., Bertolino, A., Zaitsev, E., Gold, B., Goldman, D., Dean, M., Lu, B. \& Weinberger, D.R. (2003) The BDNF val66met polymorphism affects activity-dependent secretion of BDNF and human memory and hippocampal function. Cell 112, 257-269.

Fanous, A.H., Neale, M.C., Straub, R.E., Webb, B.T., O'Neill, A.F. Walsh, D. \& Kendler, K.S. (2004) Clinical features of psychotic disorders and polymorphisms in HT2A, DRD2, DRD4, SLC6A3 (DAT1), and BDNF: a family based association study. Am J Med Genet B Neuropsychiatr Genet 125, 69-78.

Folstein, S.E. \& Rosen-Sheidley, B. (2001) Genetics of autism: complex aetiology for a heterogeneous disorder. Nat Rev Genet 2, 943-955.

Fujimura, H., Altar, C.A., Chen, R., Nakamura, T., Nakahashi, T., Kambayashi, J., Sun, B. \& Tandon, N.N. (2002) Brain-derived neurotrophic factor is stored in human platelets and released by agonist stimulation. Thromb Haemost 87, 728-734.

Fumagalli, F., Molteni, R., Roceri, M., Bedogni, F., Santero, R., Fossati, C., Gennarelli, M., Racagni, G. \& Riva, M.A. (2003) Effect of antipsychotic drugs on brain-derived neurotrophic factor expression under reduced $\mathrm{N}$-methyl-D-aspartate receptor activity. J Neurosci Res 72, 622-628.

Hashimoto, K., Iwata, Y., Nakamura, K., Tsujii, M., Tsuchiya, K.J., Sekine, Y., Suzuki, K., Minabe, Y., Takei, N., Iyo, M. \& Mori, N. (2006) Reduced serum levels of brain-derived neurotrophic factor in adult male patients with autism. Prog Neuropsychopharmacol Biol Psychiatry 30, 1529-1531.

Horvath, S., Xu, X. \& Laird, N.M. (2001) The family based association test method: strategies for studying general genotype-phenotype associations. Eur J Hum Genet 9, 301-306.

Ingram, J.L., Stodgell, C.J., Hyman, S.L., Figlewicz, D.A., Weitkamp, L.R. \& Rodier, P.M. (2000) Discovery of allelic variants of HOXA1 and HOXB1: genetic susceptibility to autism spectrum disorders. Teratology 62, 393-405.

Katoh-Semba, R., Wakako, R., Komori, T., Shigemi, H., Miyazaki, N., Ito, H., Kumagai, T., Tsuzuki, M., Shigemi, K., Yoshida, F. \& Nakayama, A. (2007) Age-related changes in BDNF protein levels in human serum: differences between autism cases and normal controls. Int J Dev Neurosci 25, 367-372.

Karege, F., Schwald, M. \& Cisse, M. (2002) Postnatal developmental profile of brain-derived neurotrophic factor in rat brain and platelets. Neurosci Lett 328, 261-264.

Lam, K.S., Aman, M.G. \& Arnold, L.E. (2006) Neurochemical correlates of autistic disorder: a review of the literature. Res Dev Disabil 27, 254-289.

Lang, U.E., Jockers-Scherubl, M.C. \& Hellweg, R. (2004) State of the art of the neurotrophin hypothesis in psychiatric disorders: implications and limitations. J Neural Transm 111, 387-411.

Lange, C. \& Laird, N.M. (2002) Power calculations for a general class of family-based association tests: dichotomous traits. Am J Hum Genet 71, 575-584.

Levitt, P. \& Campbell, D.B. (2009) The genetic and neurobiologic compass points toward common signaling dysfunctions in autism spectrum disorders. J Clin Invest 119, 747-754.

Lommatzsch, M., Zingler, D., Schuhbaeck, K., Schloetcke, K., Zingler, C., Schuff-Werner, P. \& Virchow, J.C. (2005) The impact of age, weight and gender on BDNF levels in human platelets and plasma. Neurobiol Aging 26, 115-123.

Lord, C., Rutter, M. \& Le Couteur, A. (1994) Autism Diagnostic Interview-Revised: a revised version of a diagnostic interview for caregivers of individuals with possible pervasive developmental disorders. J Autism Dev Disord 24, 659-685.

Lord, C., Risi, S., Lambrecht, L., Cook, E.H. Jr, Leventhal, B.L., DiLavore, P.C., Pickles, A. \& Rutter, M. (2000) The Autism Diagnostic Observation Schedule-Generic: a standard measure of social and communication deficits associated with the spectrum of autism. J Autism Dev Disord 30, 205-223.

Mattson, M.P., Maudsley, S. \& Martin, B. (2004) BDNF and 5-HT: a dynamic duo in age-related neuronal plasticity and neurodegenerative disorders. Trends Neurosci 27, 589-594. 


\section{Correia et al.}

Mercader, J.M., Ribases, M., Gratacos, M., Gonzalez, J.R., Bayes, M., de Cid, R., Badia, A., Fernandez-Aranda, F. \& Estivill, X. (2007) Altered brain-derived neurotrophic factor blood levels and gene variability are associated with anorexia and bulimia. Genes Brain Behav 6, 706-716.

Miyazaki, K., Narita, N., Sakuta, R., Miyahara, T., Naruse, H., Okado, N. \& Narita, M. (2004) Serum neurotrophin concentrations in autism and mental retardation: a pilot study. Brain Dev 26 292-295.

Nelson, K.B., Grether, J.K., Croen, L.A., Dambrosia, J.M., Dickens, B.F., Jelliffe, L.L., Hansen, R.L. \& Phillips, T.M. (2001) Neuropeptides and neurotrophins in neonatal blood of children with autism or mental retardation. Ann Neurol 49, 597-606.

Nelson, P.G., Kuddo, T., Song, E.Y., Dambrosia, J.M., Kohler, S., Satyanarayana, G., Vandunk, C., Grether, J.K. \& Nelson, K.B. (2006) Selected neurotrophins, neuropeptides, and cytokines: developmental trajectory and concentrations in neonatal blood of children with autism or Down syndrome. Int J Dev Neurosci 24, 73-80.

Neves-Pereira, M., Mundo, E., Muglia, P., King, N., Macciardi, F. \& Kennedy, J.L. (2002) The brain-derived neurotrophic factor gene confers susceptibility to bipolar disorder: evidence from a familybased association study. Am J Hum Genet 71, 651-655.

Nishimura, K., Nakamura, K., Anitha, A. et al. (2007) Genetic analyses of the brain-derived neurotrophic factor (BDNF) gene in autism. Biochem Biophys Res Commun 356, 200-206.

O'Connell, J.R. \& Weeks, D.E. (1998) PedCheck: a program for identification of genotype incompatibilities in linkage analysis. Am $J$ Hum Genet 63, 259-266.

Pardo, C.A. \& Eberhart, C.G. (2007) The neurobiology of autism. Brain Pathol 17, 434-447.

Pearse, A.G. (1980) The common peptides and the cytochemistry of their cells of origin. Basic Appl Histochem 24, 63-73.

Perry, E.K., Lee, M.L., Martin-Ruiz, C.M., Court, J.A., Volsen, S.G., Merrit, J., Folly, E., Iversen, P.E., Bauman, M.L., Perry, R.H. \& Wenk, G.L. (2001) Cholinergic activity in autism: abnormalities in the cerebral cortex and basal forebrain. Am J Psychiatry 158, 1058-1066.

Philippi, A., Tores, F., Carayol, J., Rousseau, F., Letexier, M., Roschmann, E., Lindenbaum, P., Benaijou, A., Fontaine, K. Vazart, C., Gesnouin, P., Brooks, P. \& Hager, J. (2007) Association of autism with polymorphisms in the paired-like homeodomain transcription factor 1 (PITX1) on chromosome 5q31: a candidate gene analysis. BMC Med Genet 8, 74 .
Ribases, M., Hervas, A., Ramos-Quiroga, J.A., Bosch, R., Bielsa, A., Gastaminza, X., Fernandez-Anguiano, M., Nogueira, M., GomezBarros, N., Valero, S., Gratacos, M., Estivill, X., Casas, M., Cormand, B. \& Bayes, M. (2008) Association study of 10 genes encoding neurotrophic factors and their receptors in adult and child attention-deficit/hyperactivity disorder. Biol Psychiatry 63, 935-945.

Stoilov, P., Castren, E. \& Stamm, S. (2002) Analysis of the human TrkB gene genomic organization reveals novel TrkB isoforms, unusual gene length, and splicing mechanism. Biochem Biophys Res Commun 290, 1054-1065.

Trajkovska, V., Marcussen, A.B., Vinberg, M., Hartvig, P., Aznar, S. \& Knudsen, G.M. (2007) Measurements of brain-derived neurotrophic factor: methodological aspects and demographical data. Brain Res Bull 73, 143-149.

Vyssotski, A.L., Dell'Omo, G., Poletaeva, I.I., Vyssotsk, D.L., Minichiello, L., Klein, R., Wolfer, D.P. \& Lipp, H.P. (2002) Longterm monitoring of hippocampus-dependent behavior in naturalistic settings: mutant mice lacking neurotrophin receptor TrkB in the forebrain show spatial learning but impaired behavioral flexibility. Hippocampus 12, 27-38.

Yu, H., Zhang, Z., Shi, Y., Bai, F., Xie, C., Qian, Y., Yuan, Y. \& Deng, L. (2008) Association study of the decreased serum BDNF concentrations in amnestic mild cognitive impairment and the Val66Met polymorphism in Chinese Han. J Clin Psychiatry 69. $1104-1111$

Zetterstrom, T.S., Pei, Q., Madhav, T.R., Coppell, A.L., Lewis, L. \& Grahame-Smith, D.G. (1999) Manipulations of brain 5-HT levels affect gene expression for BDNF in rat brain. Neuropharmacology 38, 1063-1073.

Zorner, B., Wolfer, D.P., Brandis, D., Kretz, O., Zacher, C., Madani, R., Grunwald, I., Lipp, H.P., Klein, R., Henn, F.A. \& Gass, P. (2003) Forebrain-specific trkB-receptor knockout mice: behaviorally more hyperactive than "depressive". Biol Psychiatry 54, 972-982.

\section{Acknowledgments}

We thank the autistic patients and their families for their collaboration. This work was supported by the grant PTDC/SAU/ GMG/64519/2006 from 'Fundação para a Ciência e Tecnologia' (FCT). C.T.C. was supported by the FCT fellowship SFRH/BD/ 16907/2004. Drs S.E., L.G. and M.G. are supported by the Health Research Board and Autism Speaks. 